Alexis V. Pinilla Díaz ${ }^{1}$

Profesor Universidad Santo Tomás

\title{
FORMACIÓN DE MAESTROS Y EDUCACIÓN A DISTANCIA. IMPACTO Y PERSPECTIVAS
}

La capacidad de cobertura del Estado colombiano, en materia de políticas públicas, ha sido abundante, pero no acertada, a lo largo del siglo XX. Ejemplo de ello es la incapacidad del sistema educativo, tanto en la educación básica y media como en la educación superior, para absorber la demanda educativa del ámbito nacional y, particularmente, de los sectores rurales alejados de los principales centros urbanos del país. Esta situación ha generado una aceleración del índice de priva- tización de la educación, manifiesto, con bastante fuerza, desde mediados del siglo XX, cuando los gobiernos conservadores del momento impulsaron y apoyaron a la empresa y a la iglesia en el proceso de creación de instituciones educativas en todos los niveles de enseñanza. En el siguiente cuadro se muestra un acercamiento a la escasa cobertura del sistema de educación superior $^{3}$, propia de la década de los 80 :

\begin{tabular}{|c|c|c|c|c|}
\hline Año & No. de bachilleres & Matrícula 1er año & Tasa de absorción & No ingresan \\
\hline 1980 & 163.370 & - & - & - \\
1981 & 175.663 & 124.521 & $76,2 \%$ & $23,8 \%$ \\
1982 & 189.686 & 122.688 & $69,8 \%$ & $30,2 \%$ \\
1983 & 206.902 & 130.793 & $68,9 \%$ & $31,1 \%$ \\
1984 & 243.648 & 138.709 & $67 \%$ & $33 \%$ \\
1985 & 273.869 & 147.003 & $60,3 \%$ & $39,7 \%$ \\
1986 & 307.342 & 155.108 & $56,6 \%$ & $43,4 \%$ \\
1987 & - & 163.213 & $53,1 \%$ & $46,9 \%$ \\
\hline
\end{tabular}

\footnotetext{
${ }^{1}$ Director de la línea de investigación Procesos históricos y contextos de la educación abierta y a distancia de la Universidad Santo Tomás. E-mail: avpinidi@usta.edu.co

${ }^{2}$ Este artículo hace parte del proyecto de investigación "Inicios de los programas de educación superior a distancia: impacto en la formación del magisterio colombiano, 1970-1992", adscrito a la Facultad de Educación, Universidad Santo Tomás. La coinvestigadora
}

de este proyecto es Fabiola Cuellar, docente de esta misma Facultad. Una versión inicial de este artículo fue presentada en la $46^{\underline{a}}$ Asamblea Mundial del International Council of Education for teaching, realizada en Santiago de Chile del 23 al 27 de julio de 2001.

${ }^{3}$ Fuente: Margarita Castro, "Estado actual y prospectiva de la educación superior a distancia", en Documentación Educativa, № 19, Bogotá, 1983. 
En cuanto al crecimiento del sector educativo privado y el público en la educación superior anotemos que, entre 1970 y 1980 , el sector educativo privado se quintuplicó (de 27 centros educativos en 1970 a 132 en el 80) mientras que el sector oficial apenas se duplicó (23 a 56 centros educativos, respectivamente) $)^{4}$. Para la década de los 80 , según algunos autores ${ }^{5}$, las instituciones públicas crecieron, entre 1981 y 1985 , en un $100 \%$ mientras que las privadas en un 425\%. Este distanciamiento del Estado sobre el control de la oferta educativa y del mismo funcionamiento de la educación, resultó demasiado traumático para el proceso de consolidación de un sistema educativo nacional a partir del cual fundamentar las bases de un Estado nacional. El fraccionamiento de la oferta educativa, ocasionado por la acción del sector privado auspiciada por el Estado, profundizó las serias fracturas de la sociedad civil colombiana y contribuyó a que el Estado y la nación se continuaran construyendo de manera paralela y aislada.

Ante la poca capacidad del Estado para responder a las demandas educativas de la población nacional emergió, a principios de la década de los 70 , en el ámbito de la educación superior, la modalidad de la educación a distancia que respondió, en su momento, a dos estrategias: por

\footnotetext{
${ }^{4}$ Icfes, "Encuentro de Universidades con Programas de Educación a Distancia Estado Actual y Prospectiva de la Educación Superior a Distancia”, Bogotá, ICFES, 1983. ${ }^{5}$ Jaime Arias, Humberto Serna y Alfonso Borrero, Universidad a distancia: del sueño a la realidad, Bogotá, FES, s.f.
}

un lado, a la descentralización de la oferta educativa y, por otro, a la democratización del sistema educativo colombiano. Los primeros intentos por descentralizar la educación superior y llevarla a las zonas más alejadas de los centros urbanos corrieron a cargo de las instituciones privadas. No obstante, de acuerdo a los diagnósticos hechos por organismos como el lcfes, si bien la modalidad a distancia contribuyó al aumento de la población inscrita en la educación superior, los niveles de calidad de la misma con respecto a la educación superior presencial eran muy bajos. Quizás esto obedece a que los primeros programas a distancia se orientaron más por la mercantilización de la oferta educativa, que por una intención democratizadora y cualificadora de la educación superior.

\section{Breves antecedentes de la educación a distancia en Colombia}

Con anterioridad a la aparición de los programas de educación superior a distancia se habían dado experiencias de formación abierta y a distancia, dentro de las cuales se pueden señalar las escuelas por correspondencia (1930), la educación por radio (finales de los 40), la televisión educativa (década de los 60) y los programas extensivos del Servicio Nacional de Aprendizaje-SENA- (característicos de los 60 y 70). Buena parte de estos programas estuvieron orientados a la cualificación de sectores marginados, política y socialmente, en materias instrumentales y técnicas. Para el caso de las escuelas por correspondencia, el mo- delo que se siguió en nuestro país fue el desarrollado por la Scranton (empresa estadounidense productora de carbón y antracita), en donde se cualificaba a los obreros en temáticas de seguridad industrial y en otras áreas técnicas.

El ambiente reformista de la década de los 30 y parte de los 40 , caracterizado por la necesidad de cualificar mano de obra para su inserción en el desarrollo industrial del país, dio origen a algunas estrategias de formación paralelas al sistema educativo formal, que resultaba insuficiente frente a las demandas de la población nacional. Dentro de estas estrategias se empezaron a articular a la educación algunos medios de comunicación, tales como la radio y, posteriormente, en la década de los 60, la televisión. La educación por radio tuvo como pionero el programa de Acción Cultural Popular, el cual se orientó a cualificar a los sectores rurales. En cuanto a la televisión educativa anotemos que en sus primeros años de existencia tuvo una amplia cobertura y aceptación ${ }^{6}$. No obstante, cuando se masificó la televisión comercial, dirigida exclusivamente al esparcimiento, la televisión educativa perdió su impulso inicial.

\footnotetext{
${ }^{6}$ Hacemos referencia aquí al Fondo de Capacitación Popular de Inravisión (en el cual colaboraron el Ministerio de Educación Nacional, el Ministerio de Comunicaciones, las Secretarías de Educación y la Universidad Pedagógica Nacional), que se inició con el proyecto "Operación Capacitación Popular" en 1966. El Fondo nació mediante el acuerdo Número 09 de junio de 1967.
} 
Pero el antecedente más cercano de la educación superior a distancia lo constituyó la extensión de los programas formativos del SENA. Esta institución, fundada en 1957, ha desarrollado sus planes de formación técnicaprofesional a través de diferentes modalidades: presencial, formación en la empresa, promoción profesional popular rural y urbana $y$, finalmente, la formación abierta y a distancia que surgió en el SENA como política de formación profesional en el año de 1983, cuando ya la institución había realizado experiencias en esta dirección desde hacía siete años. Así mismo, en 1976, por petición del gobierno nacional, se conformó el Programa de Formación Profesional a través de medios masivos de comunicación, que luego se llamó Centro de Formación a Distancia (Cefad).

\section{El contexto de la educación superior a distancia en el período 1970-1992}

Uno de los principales problemas que afectaban a la educación colombiana, para inicios de la década de los 70 , era la escasa cobertura del sistema educativo. $\mathrm{Si}$ las poblaciones marginales urbanas tenían muy pocas posibilidades de acceder al sistema educativo en su primeros niveles, la situación en el sector rural era aún más crítica. Según Aline Helg, si bien las políticas educativas de los 70 "permitieron un aumento de la matrícula total, no disminuyeron la brecha entre las oportunidades educativas del campo y de las ciudades. En 1970 todavía cerca del $40 \%$ de los niños en edad esco- lar de las zonas rurales no asistía a la escuela, mientras en las zonas urbanas sólo el $22 \%$ no asistía"7.

En materia de educación superior ocurrieron dos fenómenos que contribuyeron al crecimiento de la oferta educativa. Por un lado, la transformación del Fondo Universitario Nacional en el actual Instituto Colombiano para el Fomento de la Educación Superior (Icfes), reorientó el sentido y la dirección de la educación
Estas estrategias conllevaron a que en 1980 Colombia fuera uno de los países del mundo con mayor número de universidades, pues contaba con 201 universidades, de las cuales 56 eran de carácter oficial (es decir el $28 \%$ ) y 145 privadas (lo que equivale al $72 \%)$. Como se anotó anteriormente, la privatización de la educación es una constante de la evolución educativa nacional desde mediados del siglo XX. Es importante anotar que si bien el porcentaje de ingreso a la uni-

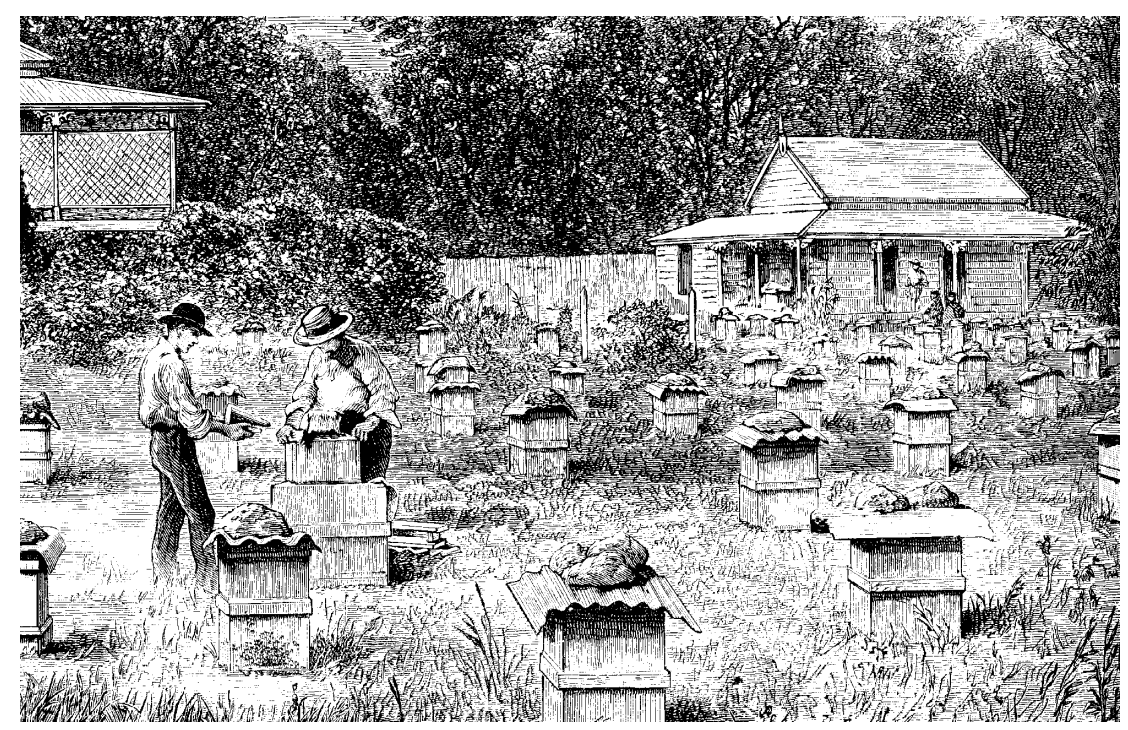

superior. De otro lado, el país recibió un fuerte apoyo de los Estados Unidos para la fundación de universidades, la mayoría de ellas provenientes de capital privado, como parte de la estrategia de frenar el avance ideológico de la revolución cubana en el continente americano.

\footnotetext{
${ }^{7}$ Aline Helg, "La educación en Colombia: 1958-1980", en Nueva Historia de Colombia, tomo IV, Bogotá, Planeta, 1998, 2ª edición, p. 150.
}

versidad creció entre 1970 y 1980 en un $278 \%$, este crecimiento no se tradujo en términos de calidad. La proliferación de instituciones universitarias y de universidades soportadas por una lógica mercantilista, antes que académica e investigativa, pone en entredicho la calidad de muchos de los programas ofrecidos para esta época.

Otra característica de la educación superior en el país es la con- 
centración de las matrículas en los grandes centros urbanos. Para 1968, por ejemplo, hay un $54 \%$ de la matrícula universitaria en Bogotá. Las ciudades de Barranquilla, Cali y Medellín representan el $26 \%$ del total de matrículas del país y sólo el $19 \%$ se distribuye en el resto del territorio nacional ${ }^{8}$. Para inicios de la década de los 80 se mantuvo la concentración de la matrícula en Bogotá en un índice aproximado al $45 \%$. Podemos anotar, siguiendo a Mariana Serrano y Ricardo Lucio, que el proceso de consolidación de las universidades colombianas en el período 1975-1990 es, en efecto, un fenómeno eminentemente urbano $^{9}$.

En este contexto la emergencia de la educación superior a distancia era algo más que un resultado previsible. La escasa capacidad de cobertura del Estado en materia educativa, la concentración de la matrícula universitaria en los grandes centros urbanos, los acelerados niveles de privatización de la educación, crearon el ambiente necesario para que algunas universidades descentralizaran la oferta educativa Ilevándola hasta los municipios mismos en que se encontraba la población. Dentro de las experiencias universitarias pioneras podemos señalar las siguientes: el programa a distancia de la Universidad de Antioquia (1972); el Programa de

${ }^{8}$ Germán Rama. El sistema universitario en Colombia, Bogotá, Universidad Nacional 1970.

${ }^{9}$ Mariana Serrano y Ricardo Lucio. La Educación Superior. Tendencias y políticas estatales, Bogotá, Universidad Nacional, 1992.

Universidad Abierta (PUA) de la Universidad Javeriana (1974), a través del cual se avanzó en la capacitación de más de 600 maestros entre 1974 y $1982^{10}$; el Instituto de Educación a Distancia de la Universidad de la Sabana (julio de 1975), a través del cual se ofrecieron cuatro programas: Administración y supervisión educativa, Licenciatura en Ciencias Sociales, Enseñanza de las bellas artes y Licenciatura en lingüística y literatura ${ }^{11}$; el Centro de Enseñanza Desescolarizada de la USTA ${ }^{12}$; el Programa de Profesionalización a Distancia de la Universidad de San Buenaventura (en 1977 se inicia con dos especializaciones: Licenciatura en investigación y educación primaria y Administración educativa y planeamiento curricular); $y$, finalmente, el Programa de actualización a Distancia para Docentes del Ministerio de Educación Nacional, con tres modalidades:

\begin{tabular}{|c|c|c|c|c|c|c|c|}
\hline $\begin{array}{c}\text { Normal } \\
\text { superior }\end{array}$ & $\begin{array}{c}\text { Normal } \\
\text { Rual }\end{array}$ & Bachiller & Comercial & Licenciat. & Otro & Sin & Total \\
\hline 580 & 375 & 260 & 17 & 3 & 63 & 690 & 1998 \\
\hline
\end{tabular}

Para inicios de la década de los 80 , la educación superior a distancia se inscribió con claridad en la política de Estado.

\footnotetext{
${ }^{10}$ La Javeriana utilizó dos medios: la televisión (con los programas "Universidad abierta" y "Educadores de hombres nuevos") y la correspondencia. A partir de 1982 el PUA se orienta a la profesionalización de maestros en ejercicio y a la formación de licenciados

${ }^{11}$ La Universidad de la Sabana implementa cuatro estrategias para sus estudiantes: por correspondencia, cursos vacacionales, tutorías y, finalmente, pruebas de eficiencia

${ }^{12}$ En 1970 la Santo Tomás inició la desescolarización de la Facultad de Filosofía y
}

profesionalización, especialización y actualización.

Uno de los proyectos de gran impacto en la formación de profesores en los inicios de los programas a distancia o desescolarizados, fue el adelantado por la Universidad Javeriana, entre 1973-1974, denominado Educadores de hombres nuevos. Con el desarrollo de este programa se intentó mostrar a los docentes algunos de los adelantos que se estaban dando en la enseñanza, tanto en el uso de los recursos técnicos y la distribución del tiempo de trabajo de los estudiantes, como sobre los métodos de socialización de las actividades escolares. La cobertura a nivel nacional (16 departamentos) en número de estudiantes (docentes en ejercicio) que tuvo este programa durante el período señalado fue de 1998, distribuidos de la siguiente manera ${ }^{13}$ :

Mediante la reforma de la educación superior de estos años, se conformó el sistema de educación postsecundaria a partir del cual se reflexionó sobre la orientación de las políticas educativas que lograran la apertura de la educación. En esta reforma de principios de los 80 se definieron las modalidades 0

\footnotetext{
Ciencias Religiosas, cuyo exito conllevó a la conformación del CED en 1976. Las estrategias de la USTA fueron dos: utilización de textos guía y tutorías.

${ }^{13}$ Fuente: Programa de Educadores de hombres nuevos, Bogotá, Icfes, 1975.
} 
niveles educativos del sistema, a saber: formación intermedia profesional, tecnológica, universitaria y, finalmente, de avanzada o postgrado. Para Roberto Salazar,

La inclusión de las modalidades intermedia profesional y formación tecnológica dentro del sistema universitario es una respuesta político jurídica ante las demandas por ingreso que permite graduar, a manera de dique, las demandas por educación superior. En este escalonamiento se establece la correlación entre la naturaleza y la duración de las carreras con las oportunidades para el empleo, así como la gradación de menor a mayor componente investigativo, como ápice se encuentra en la formación avanzada y de postgrado; e introduce, aunque de manera dosificada, la formación humanística en todos los niveles de la formación possecundaria, alcanzando su mayor despliegue en la universitaria ${ }^{14}$.

Dentro de las reformas propuestas para la organización y funcionamiento de la educación superior, se hizo evidente la necesidad de promover y facilitar la apertura del sistema educativo a mayor cantidad de población nacional. En esta medida se propuso una reglamentación y reorientación de la educación superior a distancia, a partir, fundamentalmente, del desarrollo del decreto ley 80 de 1980. Según Margarita Castro, entre 19791980 el Icfes orientó su labor a la identificación de criterios que permitieran la reglamentación de

\footnotetext{
${ }^{14}$ Roberto Salazar, "Aproximaciones arqueológicas y perspectivas de la educación superior a distancia en Colombia", en Conferencia Internacional de educación a Distancia (Memorias), MEN, Icfes, Bogotá, 1999, p. 62.
}

los programas de educación a distancia existentes en las universidades y el fomento de ellos en las instituciones que ofrecieran garantía para su buen desarrollo. En 1982, tras un análisis de los programas a distancia se mostró que el número de alumnos era de 9.941, de los cuales el $98,8 \%$ estaba compuesto por docentes o administrativos educativos. El 1,2\% restante son enfermeras que cursan el último año para obtener la licenciatura. En este aspecto empieza a verse que "es notoria una mayor cantidad de programas en el área de las Ciencias de la Educación. Y dentro de esta área es importante notar que los más frecuentes son los programas de humanidades y ciencias sociales"15.

A través del decreto 2412 de 1982, en el cual trabajaron Galo Burbano (Icfes), Liliam Suárez Melo (Secretaría Jurídica de la Presidencia) y el MEN Jaime Arias Ramírez, se reglamentó el desarrollo e inspección de la educación superior abierta y a distancia y se creó el Programa Nacional de Educación Superior Abierta y a Distancia. En octubre de 1982, se creó la subdirección de tecnología educativa, la cual se fue transformando paulatinamente en la subdirección de educación a distancia, y se encargó a Margarita de Ramírez de su dirección. Para Jaime Arias, "la tarea era monumental y por qué no, angustiosa. España esperó más de cinco años antes de comenzar a dictar cursos; Inglaterra había destinado casi una década a la planeación y construc-

\footnotetext{
${ }^{15}$ Margarita Castro, op cit., p. 135.
}

ción de la infraestructura docente y nosotros los colombianos, por razones justificadas teníamos que recorrer el camino en pocos meses"16. Es importante enunciar que si bien la reglamentación sobre la educación a distancia data de principios de los 80 , ya desde la segunda mitad de los 70 el gobierno había proyectado la organización del Programa Nacional de educación a distancia.

En un primer momento el Icfes encargó esta tarea a la Universidad de Antioquia, la cual tenía varios años de experiencia a nivel regional en educación a distancia. El proyecto de universidad desescolarizada intentaba vincular la utilización de la correspondencia y emisiones radiales contrastándolo con el sistema presencial de la misma universidad. En este sentido el proyecto, en su fase experimental, buscó tres objetivos principales, a saber: comparar la efectividad interna de un sistema desescolarizado (vía correspondencia y radio) con el sistema escolarizado formal de la Universidad de Antioquia; detectar las deficiencias de dicho sistema y, finalmente, adelantar un estudio de costos por estudiante y una proyección del mismo en el sistema desescolarizado.

En esta primera etapa la desescolarización se entendió como la "utilización de la instrucción programada como técnica de la instrucción especialmente en la enseñanza de ciertas unidades que a juicio de los programadores tengan más probabilidad de ser asimiladas a través de este me-

\footnotetext{
${ }^{16}$ Jaime Arias, Humberto Serna y Alfonso Borrero, op. cit., p. 23.
} 
dio"17. Dentro de los objetivos del proyecto se puede ver que todos hacen relación a la necesidad de comparar (diferentes ítems y niveles de rendimiento) la propuesta desescolarizada con la formal, es decir, que en esta primera etapa el proyecto antes que surgir de manera autónoma lo hace teniendo como referente la universidad presencial formal.

Otra característica importante es que la educación a distancia surge imbuida del ambiente de la tecnología educativa en el país. En este sentido se consideró que la aplicación de la tecnología educativa afectaba positivamente el rendimiento del grupo desescolarizado neutralizando las desventajas de éste con respecto al escolarizado y, además, que la tecnología utilizada en el proyecto desescolarizado aumentaba los niveles de motivación y satisfacción de los estudiantes vinculados al mismo. La tecnología educativa a principios de los 70 , y su inserción en la organización y proyección de la educación a distancia, fue concebida como, "el desarrollo, aplicación y evaluación de sistemas, técnicas y medios de acuerdo con objetivos formulados previamente y que tienen como fin el hacer más efectivo el aprendizaje. Esta concepción no se limita a la utilización de medios o de máquinas complejas (hardware); se trata, principalmente, del diseño de sistemas en los que dichos instrumentos adquieran sentido para el educador y el educando" 18 .

${ }^{17}$ Icfes, Proyecto Nacional de Educación Superior a Distancia, Icfes, Bogotá, 1975 , p. 53.

${ }_{18}$ Pontificia Universidad Javeriana, Programa Educadores de Hombres Nuevos. In-

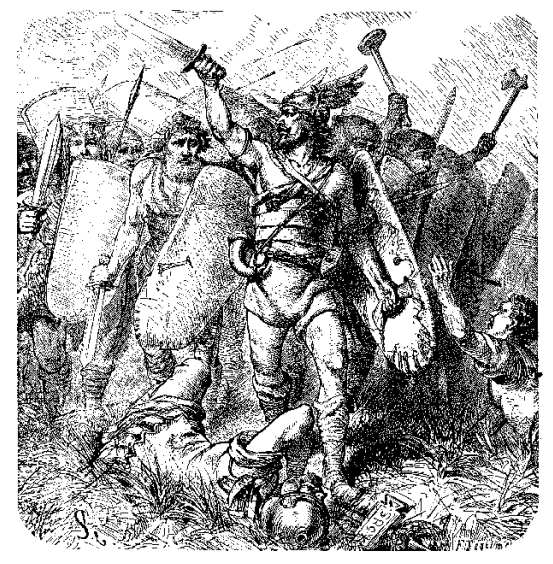

Se puede anotar, entonces, que los planes orientados a la formación/profesionalización de los maestros tenían como una característica central la difusión de la tecnología educativa como una estrategia pedagógica y metodológica de especial importancia para el desarrollo educativo nacional. En la segunda etapa del proyecto (dirigida por Jairo Arboleda Toro) se decidió fortalecer tres programas académicos: matemáticas, sicología y español, por considerar que eran requisito de todos los estudiantes de educación y por ser materias "menos difíciles de tratar mediante las técnicas de instrucción programada"19.

En esta coyuntura también tuvo lugar la creación de la Unisur, la cual se constituyó en la primera universidad a distancia de carácter estatal. Para Hernando Bernal, primer rector de la Unisur, el sistema a distancia no consiste únicamente en la vinculación de medios de comunicación a los

forme 1972, Facultad de educación, Pontificia Universidad Javeriana, Icfes, 1975, p. 262.

${ }^{19}$ Ibíd., p. 96. procesos de aprendizaje, sino que debe ser entendido como una modalidad que trata de acercar las facilidades educativas hacia los sitios donde vive y trabajan los estudiantes, con el fin de enseñarlos a aprender en forma individual. El sistema de educación superior a distancia surge como una respuesta a una crisis del sistema universitario formal que se evidencia en: desfase de los programas universitarios con los requerimientos de la sociedad actual, desajustes entre la preparación universitaria y la estructura ocupacional, ineficiencia de los métodos tradicionales, carencia de recursos humanos, materiales y físicos para un desarrollo integral, escasa cobertura de algunos programas y deserción estudiantil y los altos costos por matrícula. Sumado a estas características, podemos indicar que algunos de los índices mostrados por el lcfes en la coyuntura de principios de los 80 que sirvieron como justificación para la organización del programa Nacional de educación a distancia, a nivel general, y para la creación de la Unisur, son los siguientes: tasa de escolari-

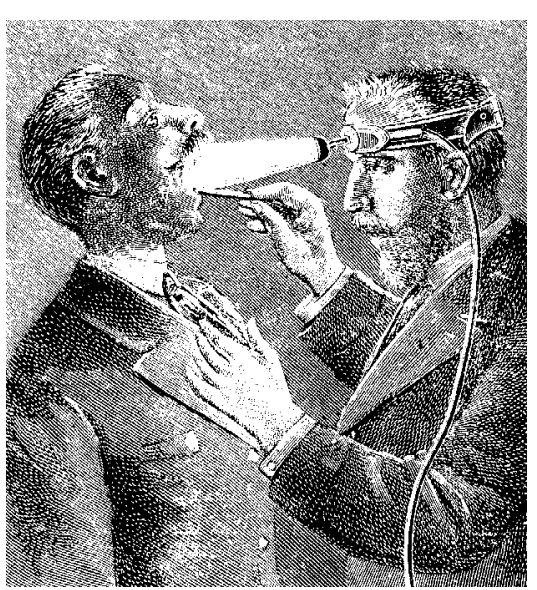


dad del 7,6\%, capacidad de absorción del sistema entre 25 y $30 \%$, concentración de $40,8 \%$ de todos los estudiantes universitarios en Bogotá, entre otros.

En esta perspectiva se pensó que Unisur podía ayudar a resolver tales problemas de acuerdo con las políticas trazadas por el gobierno. "Unisur se programa a partir de la comunidad y como una respuesta concreta a las aspiraciones populares, esencialmente de los sectores marginados del país. Unisur juega el papel de la experiencia piloto en la educación superior abierta y a distancia, de tal manera que permita conocer y evaluar una forma de educación a distancia para la Colombia marginada y popular en un proceso de investigación/acción y en términos del uso sistemático de medios reforzado por la comunicación interpersonal y la acción dinámica de grupos" 20 .

Los programas académicos con que inicia sus labores la Unisur son los siguientes: Ingeniería de alimentos, Administración de empresas, Ciencias sociales y desarrollo comunitario y Ciencias de la salud. En su estructura se muestran, entre otras, las figuras de los CREADS (Centros Regionales de Educación Superior Abierta y a Distancia) y los CIPAS (Centros de Interacción y Participación Académica y Social). Los Creads se concibieron como unidades operativas o centros de recursos educativos, en el ámbito social y regional y funcionaron como núcleos de interacción

20 Unisur, Unisur y la educación superior abierta y a distancia, Bogotá, MEN, Icfes, 1983, p. 22. y convivencia social. Los Cipas, por su parte, debían responder a criterios políticos administrativos (descentralización de la administración y desconcentración de servicios), criterios socio económicos (participación democrática en la promoción, organización y dotación de los centros regionales) y a criterios socio educativos (participación responsable en la utilización de los servicios y recursos educativos de los centro regionales). En últimas, la apertura de los centros regionales debe tener en cuenta las posibilidades reales de funcionamiento en la comunidad y de propender por la promoción de la comunidad local y regional en la que éste se encuentre. En cuanto a los tutores se anotó que éstos son agentes educativos que promueven, facilitan y mantienen los procesos de comunicación necesarios para contribuir al perfeccionamiento del sistema (aumento de la calidad del aprendizaje y la realización personal del estudiante).

De manera general, es posible anotar que las carreras privilegiadas para la universidad a distancia fueron las de tipo técnico y tecnológico que respondiesen a las necesidades regionales $y$, fundamentalmente, a las exigencias de la economía nacional. Con lo anterior queremos aclarar que la organización de la educación a distancia responde a los contextos y ambientes socio económico en los cuáles ésta se encuentra inscrita. Para las décadas de los 70 y 80 hubo un cambio significativo en la composición de la actividad económica en el país, en la cual se empezaron a consolidar la industria manufacturera, el transporte, el sector finan- ciero, el de comunicaciones y los servicios públicos. Estos sectores pasaron de representar el $23 \%$ de la actividad económica, en la década de los 40 , a cerca del $40 \%$ en los inicios de los $80^{21}$. Podría pensarse que este cambio en la composición de la actividad económica nacional, con sus implicaciones en el plano regional, reorientó las prioridades educativas y formativas difundidas por el gobierno.

En este contexto, podemos anotar que la orientación de la universidad a distancia propendía porque no se presentara una concentración de las carreras liberales (derecho, medicina, economía, etc.) como en la universidad presencial, sino que los programas académicos respondiesen, directamente, a las necesidades, características y demandas de las regiones. No obstante, junto a las carreras técnicas, también sobresalieron los programas ofrecidos para cualificar el magisterio y profesionalizar a gran cantidad de docentes en ejercicio que no tenían título de licenciatura. Así, uno de los primeros impactos que ha tenido desde sus inicios la educación a distancia en la formación del magisterio tiene que ver con planes de profesionalización regional de docentes. A continuación se muestra la evolución de la matrícula en programas de educación a distancia ${ }^{22}$.

\footnotetext{
${ }^{21}$ José Antonio Ocampo, Joaquín Bernal, Mauricio Avella y María Errázuriz, "La consolidación del capitalismo moderno", en José Antonio Ocampo (compilador), México, Editorial Siglo XXI, 1988.

${ }^{22}$ Fuente: Icfes, Aproximaciones a las estadísticas de la educación superior abierta y a distancia, Bogotá, Icfes, UNAD, 1998.
} 
Matrículas en programas de licenciatura en educación a distancia

\begin{tabular}{|c|c|c|c|c|c|}
\hline Año & $\begin{array}{c}\text { Total matrículas } \\
\text { en educación }\end{array}$ & $\begin{array}{c}\text { Hombres } \\
\text { educación a distancia }\end{array}$ & $\begin{array}{c}\text { Mujeres } \\
\text { educación a distancia }\end{array}$ & Total & $\begin{array}{c}\text { \% respecto al total } \\
\text { de matrículas }\end{array}$ \\
\hline 1984 & 14332 & 3200 & 8672 & 11872 & 82,83 \\
\hline 1985 & 22617 & 2064 & 6424 & 8488 & 37,52 \\
\hline 1986 & 31003 & 3254 & 8352 & 11606 & 37,43 \\
\hline 1987 & 25220 & 3106 & 9325 & 12431 & 49,29 \\
\hline 1988 & 21129 & 2523 & 7766 & 10289 & 48,69 \\
\hline 1989 & 18711 & 2246 & 5839 & 8085 & 43,20 \\
\hline 1990 & 15651 & 1560 & 4782 & 6342 & 40,52 \\
\hline 1991 & 17616 & 1924 & 5035 & 6959 & 39,50 \\
\hline 1992 & 13829 & 1756 & 3795 & 5551 & 40,14 \\
\hline 1993 & 16315 & 2087 & 5954 & 8041 & 49,28 \\
\hline 1994 & 23535 & 2456 & 5474 & 7930 & 33,69 \\
\hline 1995 & 29077 & 2779 & 7090 & 9869 & 33,94 \\
\hline
\end{tabular}

Como se puede notar, en la evolución de los programas a distancia durante de década de los 80 hubo una fuerte presencia de programas de licenciatura. Al parecer el rasgo de que los programas a distancia en sus orígenes se orientaran más a programas formadores de docentes (o planes de profesionalización de docentes en ejercicio), se transmitió a través de toda la década de los 80 y continuó siendo significativo en la última década del siglo XX. Contrario a lo que sucede en la modalidad presencial, en donde el número de estudiantes que optan por los programas formadores de docentes disminuye año tras año, en la educación a distancia continúan siendo más atractivos para los estudiantes los programas de licenciatura.

La década de los 90 empieza, en materia de educación superior, con un ambiente reformista evidenciado en la expedición de la ley 30 de 1992 que, a grandes rasgos, tiene tres elementos de gran importancia, a saber: la suprema inspección y vigilancia de la educación, la autonomía universitaria y, finalmente, la creación del Sistema Nacional de Acreditación, a través del cual se regula y controla la calidad de la educación superior. No obstante, a lo largo de esta década también ha resultado incontrolable la explosión de la oferta educativa por parte de instituciones que no reúnen los requisitos mínimos para desempeñar dicha tarea.

Debido a lo anterior, durante los últimos años de la década de los 90 se ha dado, en buena parte, un proceso de crecimiento continuo de la población universitaria que, si bien no significa que la cobertura de la educación superior tenga los niveles deseables para un Estado social de derecho, sí se constituye en una cifra importante para los análisis sobre la evolución de la educación superior en el país. Según datos del Icfes ${ }^{23}$, en 1960 existían 29 instituciones de educación superior en Colombia, de las cuales 12 se ubicaban en Bogotá, cifra que en 1980 asciende a 201 en todo el país y 74 en la capital. De igual forma entre 1980 y 1997 se crean, en la ciudad de Bogotá, 20 instituciones más de educación superior. En cuanto a la población estudiantil matriculada en educación superior para el año de 1960 fue de 23.013 estudiantes, cifra que pasó a 768.321 individuos en 1997 de los cuales el $41,1 \%$ se concentra en la ciudad de Bogotá.

En lo que tiene que ver con los programas educativos de mayor interés a nivel universitario en Bogotá, se puede notar que existe una mayor preferencia en la población universitaria por las carreras de Economía, Contadu-

${ }^{23}$ Tomado de, "Estadísticas de la educación superior", en sitio web del Icfes (www.icfes.gov.co). 
ría y Ciencias de la Administración $(30,9 \%$ del total de la matrícula.); seguidas de Ingeniería, Arquitectura, Urbanismo y afines $(27,1 \%)$; en un nivel intermedio la tendencia está en las Ciencias de la Educación (41.484 estudiantes, lo cual corresponde al $13,1 \%$ del total de la población universitaria en la ciudad), Ciencias Sociales $(13,1 \%)$ y Ciencias de la Salud (8,4\%); y, finalmente, en los niveles menos escogidos por los estudiantes encontramos Bellas Artes (3,9\%), Agronomía, Veterinaria y afines $(1,6 \%)$, Matemáticas y Ciencias Naturales $(1,1 \%) y$, en el último lugar, las Humanidades y Ciencias Religiosas $(0,7 \%)$. Otro elemento interesante de señalar es el volumen de población universitaria en el sector privado y en el público, en donde se presentan las siguientes cifras: del total de 315.681 estudiantes universitarios de Bogotá, 265.723 se encuentra en el sector privado mientras el restante 49.958 está en las universidades públicas (es decir, un $84,17 \%$ frente a $15,83 \%$, respectivamente).

En lo relacionado con la educación superior abierta y a distancia para el año de 1997 se ofrecían en Colombia 113 programas de pregrado, 21 especializaciones y 1 maestría. La matrícula en el primer semestre de 1997 en los programas de educación a distancia representa el $10 \%$ de las matrículas totales de la educación superior, cifra que se ha mantenido desde principios de la década de los 90 hasta el mo- mento. Es importante destacar que dentro de los intereses profesionales continúan sobresaliendo las ciencias de la educación (con un $58,2 \%$ de la matrícula total), frente a lo cual algunos autores anotan que "a pesar del interés gubernamental de que la educación a distancia sirviese especialmente para promover programas tecnológicos y atender campos del conocimiento distintos a aquellos en los que existe una buena oferta por parte de instituciones presenciales, el área de mayor ofer-

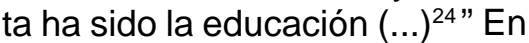
la tabla siguiente ${ }^{25}$ se muestran las estadísticas sobre el número de estudiantes matriculados por carrera en educación superior a distancia en el año de 1996:

\section{Alumnos matriculados por programa en educación a distancia en 1996}

\begin{tabular}{|l|c|c|c|}
\hline Programa & Sector Oficial & Sector Privado & Total \\
\hline Agronomía, veterinaria y afines & 1408 & 1242 & 2650 \\
\hline Ciencias de la Educación & 23519 & 35284 & 58803 \\
\hline Ciencias de la salud & 582 & 302 & 884 \\
\hline Ciencias sociales, Derecho y Política & 2118 & 2804 & 4922 \\
\hline Economía, Administración y Contaduría & 19866 & 6701 & 26567 \\
\hline Ingeniería, Arquitectura y Urbanismo & 5424 & 8496 & 13920 \\
\hline Matemáticas y Ciencias naturales & 1336 & 0 & 1336 \\
\hline Totales & $\mathbf{5 4 2 5 3}$ & $\mathbf{5 4 8 2 9}$ & $\mathbf{1 0 9 0 8 2}$ \\
\hline
\end{tabular}

Como hemos visto, desde sus inicios en 1970, los programas a distancia han tenido mayor preferencia por el campo de la educación. Sin importar que en principio la estrategia se orientaba a la cualificación de sujetos en el campo técnico instrumental, a lo largo de las décadas de los 80 y los 90 la proporción de maestros en ejercicio que accedieron a esta modalidad fue creciente. Así mismo, cerrando el siglo XX, los programas de licenciatura continúan siendo la principal elección de aquellos que optan por la educación a distancia. Si reconocemos históricamente el impacto
${ }^{24}$ Luis José González, Amaury Lora y Luis Alberto Malagón, La educación superior a distancia en Colombia, Bogotá, Icfes, 2000 , p. 26.

${ }^{25}$ Fuente: Icfes, Aproximaciones a las estadísticas de la educación superior abierta y a distancia, Bogotá, Icfes, UNAD, 1998. 
que los programas a distancia han tenido en la formación de maestros a nivel regional, es necesario contribuir en la promulgación y desarrollo de políticas, planes, programas y proyectos, con el fin de cualificar, permanentemente, el ejercicio docente, tanto a nivel regional como local. De igual forma, es interesante tener en cuenta que, por lo menos en lo concerniente a la formación de docentes en el país, la educación a distancia continúa siendo una posibilidad muy fuerte de distribución de capital cultural ${ }^{26}$. Si tenemos en cuenta que los docentes son multiplicadores de sus experiencias universitarias (sociales, políticas y cognitivas), a través de su práctica pedagógica-profesional, entonces resulta bastante significativo el impacto que tiene la formación que se imparte en programas a distancia, al interior de las diferentes regiones colombianas. El reconocimiento de esta potencialidad de la formación de docentes a distancia sería un elemento estratégico para la reformulación de políticas de formación de maestros por parte del gobierno. No obstante, esto requeriría de la existencia de una dirigencia educativa que actuara siguiendo los designios de la investigación y la academia, y no las diatribas burocráticas y clientelistas de nuestra débil democracia.

\section{Bibliografía}

ArIAS, Jaime; SernA, Humberto; Borrero, Alfonso. Universidad a distancia: del sueño a la realidad, Bogotá, FES, s.f.

CASTRO, Margarita. "Estado actual y prospectiva de la educación superior a distancia", en Documentación Educativa, № 19, Bogotá, 1983.

GonzÁLEZ, Luis José; LoRA, Amaury; MALAGÓn, Luis Alberto. La educación superior a distancia en Colombia, Bogotá, Icfes, 2000.

HeLg, Aline. "La educación en Colombia: 1958-1980", en Nueva Historia de Colombia, tomo IV, Bogotá, Planeta, 1998, 2ª edición.

Holmberg, Borje. Educación a distancia: situación y perspectivas, Buenos Aires, Kapeluz, 1985.

ICFES. Aproximaciones a las estadísticas de la educación superior abierta y a distancia, Bogotá, Icfes, UNAD, 1998.

ICFES. "Encuentro de Universidades con Programas de Educación a Distancia Estado Actual y Prospectiva de la Educación Superior a Distancia”, Bogotá, ICFES, 1983.

ICFES. Proyecto Nacional de Educación Superior a Distancia, Icfes, Bogotá, 1975, p. 53.
Ocampo, José Antonio; Bernal, Joaquín; Avella, Mauricio; Errázuriz, María. "La consolidación del capitalismo moderno", en José Antonio Ocampo (compilador), México, Editorial Siglo XXI, 1988.

Pontificia Universidad Javeriana. Programa Educadores de Hombres Nuevos. Informe 1972, Facultad de educación, Facultad de Educación, Pontificia Universidad Javeriana, Icfes, 1975.

RAMA, Germán. El sistema universitario en Colombia, Bogotá, Universidad Nacional 1970.

SALAZAR, Roberto. "Aproximaciones arqueológicas y perspectivas de la educación superior a distancia en Colombia", en Conferencia Internacional de educación a Distancia (Memorias), MEN, Icfes, Bogotá, 1999.

SerRano, Mariana; Lucio, Ricardo. La Educación Superior. Tendencias y políticas estatales, Bogotá, Universidad Nacional, 1992.

UNISUR. Unisur y la educación superior abierta y a distancia, Bogotá, MEN, Icfes, 1983.

\footnotetext{
${ }^{26}$ La idea de la educación a distancia como forma de distribución de capital cultural, la debo al profesor Alvaro Moreno, sociólogo de la Universidad Nacional.
} 